\title{
Preconditioning the uterine unfolded protein response maintains non-apoptotic Caspase 3-dependent quiescence during pregnancy
}

\author{
Judith Ingles ${ }^{1}$, Arren Simpson², Chandrashekara Kyathanahalli ${ }^{3}$, Prashanth Anamthathmakula ${ }^{3}$, Sonia Hassan ${ }^{1,3,4}$,
} Pancharatnam Jeyasuria ${ }^{1,3,4}$ and Jennifer C. Condon ${ }^{1,3,4}$

\begin{abstract}
The prevention of apoptotic caspase 3 activation through biological preconditioning, mediated through the modulation of the unfolded protein response has been demonstrated to ameliorate multiple pathophysiologies. The maintenance of non-apoptotic caspase 3 activity by the unfolded protein response within the pregnant uterus has previously been proven to be critical in inhibiting uterine myocyte contractility during pregnancy. Here we report that the pregnant uterus utilizes an unfolded protein response-preconditioning paradigm to conserve myometrial caspase 3 in a non-apoptotic state in order to effectively inhibit uterine contractility thereby preventing the onset of preterm labor. In the absence of appropriate endogenous preconditioning during pregnancy, uterine caspase 3 is transformed from a non-apoptotic to an apoptotic phenotype. Apoptotic caspase 3 activation results in the precocious triggering of local uterine inflammatory signaling and prostaglandin production, consequently resulting in an increased incidence of preterm birth. These findings represent a paradigm shift in our understanding of how preconditioning promotes the maintenance of uterine non-apoptotic caspase 3 action during pregnancy preventing the onset of premature uterine contraction and therefore defining the timing of the onset of labor.
\end{abstract}

\section{Introduction}

Activation of caspase 3 (CASP3) is typically a hallmark of cellular apoptosis or programmed cell death. Conversely, within the pregnant uterine myocyte our laboratory has established that CASP3 activation via the unfolded protein response (UPR) is completely nonapoptotic in nature and plays a critical role in the regulation of uterine myocyte quiescence ${ }^{1,2}$. While the tocolytic action of myometrial CASP3 has been highly characterized, it remains elusive as to how the uterus

Correspondence: Jennifer C. Condon (jcondon@med.wayne.edu) 'Department of Physiology, School of Medicine, Wayne State University, Detroit, MI, USA

2Department of Biology, University of Detroit Mercy, Detroit, MI, USA Full list of author information is available at the end of the article.

Edited by A. Stephanou capacitates active CASP3 in a non-apoptotic state during pregnancy. A growing body of evidence suggests that nonapoptotic CASP3 action can be maintained through the process of cellular preconditioning ${ }^{3-5}$. Throughout pregnancy, it is well established that the uterus experiences various biochemical and physiological stimuli that have been shown to trigger the UPR in other biological systems. For example, following fertilization the myometrium experiences hyperplasia ${ }^{6}$, hypertrophy ${ }^{7}$, hypoxia $^{8}$, hormone fluctuations ${ }^{9}$, inflammation ${ }^{10}$, and mechanical stretch $^{11}$ all of which have been demonstrated to activate the UPR. Subsequently, we hypothesize that incremental endoplasmic reticulum stress insults experienced by the uterus during gestation act to capacitate the myometrium to withstand additional more severe stressors, while

\section{(c) The Author(s) 2018}

(c) (i) Open Access This article is licensed under a Creative Commons Attribution 4.0 International License, which permits use, sharing, adaptation, distribution and reproduction cc) in any medium or format, as long as you give appropriate credit to the original author(s) and the source, provide a link to the Creative Commons license, and indicate if changes were made. The images or other third party material in this article are included in the article's Creative Commons license, unless indicated otherwise in a credit line to the material. If material is not included in the article's Creative Commons license and your intended use is not permitted by statutory regulation or exceeds the permitted use, you will need to obtain permission directly from the copyright holder. To view a copy of this license, visit http://creativecommons.org/licenses/by/4.0/. 
maintaining the tocolytic action of non-apoptotic CASP3, thus preventing premature uterine contractility.

Classically, CASP3 activity has been linked to the execution of cellular apoptosis through proteolytic cleavage of DNA repair molecules, such as poly ADP-ribose polymerase (PARP), resulting in internucleosomal cleavage and fragmentation of DNA. In the myometrium however, UPR-dependent activation of dna damage inducible transcript 3 (GADD153) has been demonstrated to maintain active non-apoptotic CASP3 at high levels throughout early and mid-gestation ${ }^{12}$. In other muscle systems such as the bladder, heart, and diaphragm nonapoptotic CASP3 has been described to have anticontractile function ${ }^{13-15}$. Similarly, we have shown in the uterus CASP3 inhibits myometrial contraction through the targeted cleavage and degradation of multiple components of the contractile architecture, i.e., connexin 43, $\alpha$-actin, and $\gamma$-actin ${ }^{1,2}$. While the tocolytic function of CASP3 is imminently important, understanding how the pregnant uterus maintains active CASP3 is equally important and may elucidate novel therapeutic targets for inhibiting preterm labor.

Biological preconditioning refers to the phenomenon in which prophylactic insults afford cellular/tissue tolerance against ensuing damaging or lethal stress insults, largely mediated through modulation of the local cellular UPR, allowing for enhanced cell survival resulting in a heightened resistance to apoptotic cell death ${ }^{16}$. In the events of neuronal ischemic preconditioning specifically, transient hypoxic episodes have been demonstrated to reduced cellular apoptosis while maintaining active non-apoptotic CASP3 expression following sustained ischemic injury ${ }^{5}$. As previously mentioned, the myometrium experiences various endogenous modalities of stress, i.e., hyperplasia, hypertrophy, hypoxia, hormonal fluctuations, and mechanical stretch without undergoing apoptosis, that have been demonstrated in other organ systems to illicit an UPR. In a pregnant mouse model, our laboratory has demonstrated that alteration of uterine adaptive UPR signals, i.e., glucose regulated protein (GRP78) and tocolytic CASP3 action significantly modifies gestational length ${ }^{1}$. Specifically, precocious exaggerated uterine stress prematurely increases adaptive GRP78 signaling, eliminating the tocolytic action of CASP3, leading to premature labor. Thus, it is the balance between uterine stress-mediated activation of non-apoptotic CASP3 and augmented adaptive UPR signaling that facilitates quiescence. Therefore, we hypothesize that normal transient stress insults may precondition the uterus and prevent contractility by increasing the capacity of the myometrium to experience ER stress in the absence of precocious increases in adaptive UPR signaling. Thereby maintaining tocolytic non-apoptotic CASP3 action and preventing premature labor.
To examine the role of endogenous pregnancydependent preconditioning on the maintenance of UPRdependent non-apoptotic CASP3 activity, we initially preconditioned the UPR of the telomerase immortalized human uterine myocyte cell line (hTERT-HM), which has previously been demonstrated to be comparable to primary myocyte cultures with functional equivalency and similar expression of $\alpha$ smooth muscle actin, oxytocin receptor, estrogen receptor $\alpha$, calponin, and caldesmon ${ }^{17}$, utilizing minor concentrations of tunicamycin (TM) and thapsigargin (Thaps), prior to the exposure of a known cytotoxic dose. These studies clearly demonstrated in vitro UPR preconditioning facilitates improved uterine myocyte cell viability preventing apoptotic consequences in the presence of elevated levels of CASP3 activation. In vivo, we utilized a novel pregnant mouse model where downstream stress-mediated UPR preconditioning effects were ablated by heightening tolerance to the gestational stresses through the administration of the chemical chaperone phenyl butyric acid (PBA). Interestingly, we observed increased apoptotic CASP3 activity within the endometrium, which lead to augmented prostanoid signaling, resulting in the onset of preterm birth in over $50 \%$ of the stressed-sub-preconditioned mice. Whereas endogenously preconditioned mice exposed to the same exogenous stress had a heighted capacity to maintain nonapoptotic CASP3-mediated quiescence, as $\sim 83 \%$ delivered at term.

\section{Results}

\section{UPR preconditioning renders CASP3 non-apoptotic in human uterine myocytes}

Prior to commencing this study, we first tested the effects of minor concentrations of stress (TM) on the activation of prosurvival, UPR and inflammatory signaling responses for the potential use as a preconditioning stimulus (Supplementary Figure S1). In the context of preconditioning, the ideal stress stimulus should capacitate prosurvival signaling, i.e., GRP78, without inducing proapoptotic responses. We observed $24 \mathrm{~h}$ of $0.1 \mu \mathrm{g} / \mathrm{ml} \mathrm{TM}$ significantly induced the GRP78 expression, in the absence of apoptosis or inflammation (Supplementary Figure S1). Specifically, the preconditioning stimulus did not increase activating transcription factor 4 (ATF4), nuclear factor kappa B (NF-kB), active CASP3, or GADD153 expression.

Subsequently, activation of the UPR, CASP3, and apoptotic indices were next examined by immunoblotting in control, preconditioned $(0.1 \mu \mathrm{g} / \mathrm{ml}, 24 \mathrm{~h} \mathrm{TM})$ and nonpreconditioned (vehicle) hTERT-HM cells, given a 0, 4, 24 and $48 \mathrm{~h}$ recovery period prior to a subsequent known cytotoxic dose of TM $(5.0 \mu \mathrm{g} / \mathrm{ml}, 1 \mathrm{~h})(\text { Fig. } 1)^{18}$. A robust activation of the UPR was observed in the levels of GRP78 and CASP3 in both the TM preconditioned and 
A

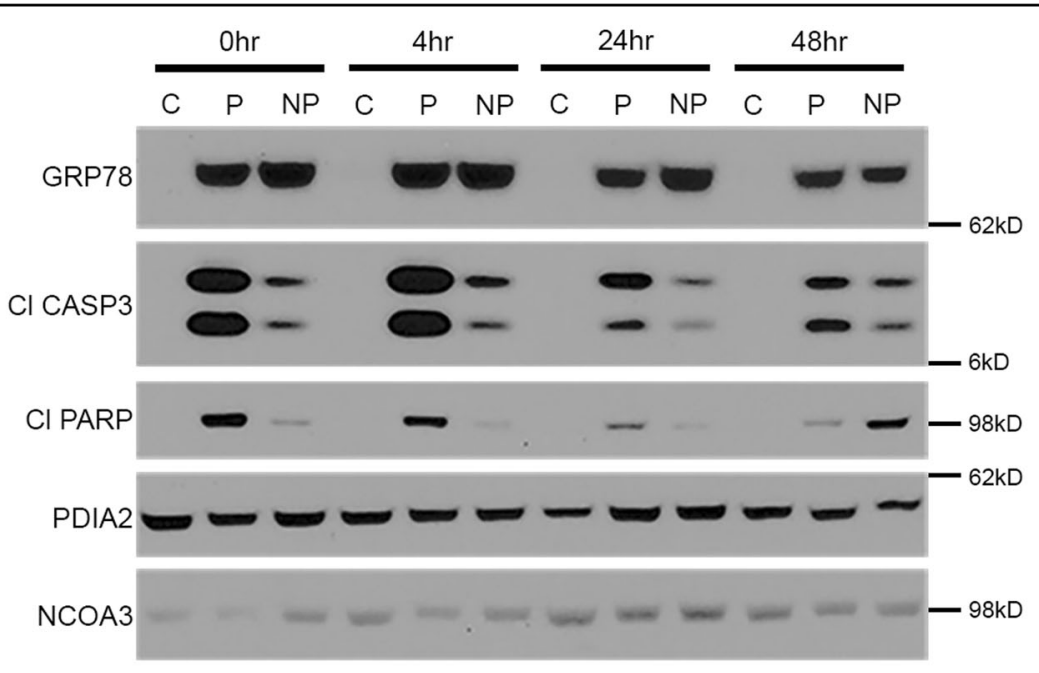

B

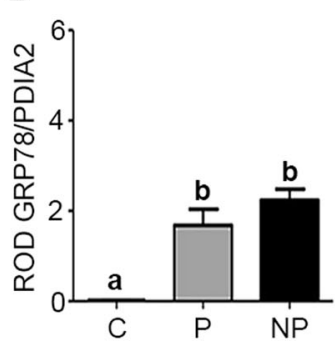

C

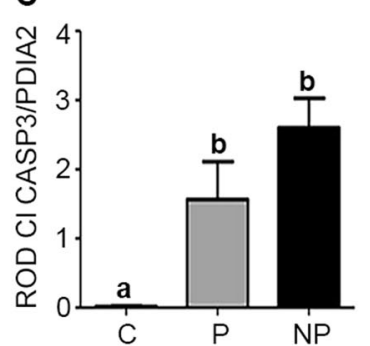

D

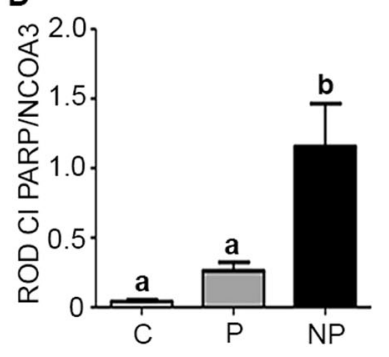

Fig. 1 UPR preconditioning renders the hTERT-HM uterine myocyte CASP3 non-apoptotic. a Elevated levels of cytoplasmic GRP78 and Cl CASP3, and nuclear CI PARP are observed in preconditioned (P) and non-preconditioned (NP) uterine myocytes as compared to controls (C) $(n=3$ per condition), when exposed to a cytotoxic dose of TM $0,4,24$, and $48 \mathrm{~h}$ post TM preconditioning. At $48 \mathrm{~h}$ to recovery there is equal activation of GRP78 (b) and CI CASP3 (c) in both P and NP uterine myocytes. In contrast, Cl PARP (d) is significantly decreased in the P versus NP cells. PDIA2 and NCOA3 are utilized as cytoplasmic and nuclear protein loading controls. A representative blot from this experiment is shown. Statistical comparisons were performed using one-way ANOVA, and subsequent Newman-Keuls multiple-comparison tests. Data labeled with different letters are significantly different from each other $(p<0.05)$

non-preconditioned cells (NP) compared to the vehicle control (Fig. 1a-c). Examination of apoptotic indices, as quantified by cleavage of the nuclear DNA repair molecule PARP, demonstrated that at all recovery time points examined CASP3 and PARP cleavage levels were equivalent, except at the $48 \mathrm{~h}$ recovery time point. With $48 \mathrm{~h}$ to recover, the uterine myocytes of the preconditioned and NP displayed equal levels of CASP3 cleavage. However remarkably the preconditioned cells had a 4-fold decrease in PARP cleavage compared to NP, demonstrating an acquired resistance to the apoptotic consequences of CASP3 activation (Fig. 1d). Decreased cell viability of the NP in comparison to control (C) and preconditioned $(\mathrm{P})$ myocytes was further validated using a trypan blue assay (Supplementary Figure S2).

To validate that the observed anti-apoptotic effects of preconditioning were not modality-dependent, we repeated our preconditioning protocol using Thaps. In which case, activation of the UPR, CASP3, and apoptotic indices were next examined by immunoblotting in control, preconditioned $(10 \mathrm{nM}, 24 \mathrm{~h}$ Thaps $)$ and nonpreconditioned (vehicle) hTERT-HM cells, given a $48 \mathrm{~h}$ recovery period prior to a subsequent known cytotoxic dose of Thaps $(250 \mathrm{nM}, 1 \mathrm{~h})$. Like TM preconditioning, activation of GRP78 was similar within the Thaps preconditioned (P) and non-preconditioned (NP) hTERT-HM cells compared to controls (C) (Supplementary Figure S3A and B). Importantly, in a manner similar to the TM preconditioning protocol Thaps-preconditioning reduced PARP cleavage by 2-fold (Supplementary Figure S3A - D) in the presence of a 25-fold increase in CASP3 activation (Supplementary Figure S3A - C) post preconditioning.

\section{UPR preconditioning inhibits inflammation in the human uterine myocyte}

To define the mediators facilitating resistance to the apoptotic consequences of CASP3, NF-kB activation in the nuclear compartment of the uterine myocyte was examined in control, preconditioned and NP exposed to TM, Thaps, or vehicle treatment. Cells were collected 


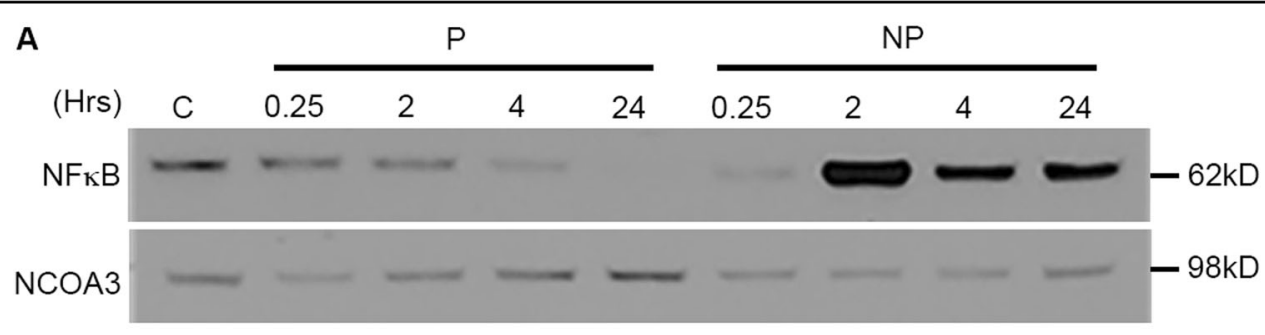

B

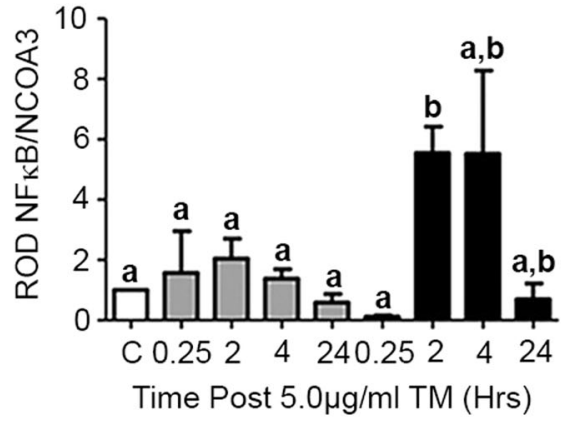

C

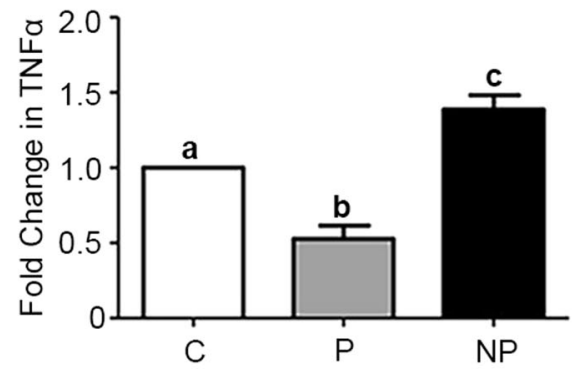

D

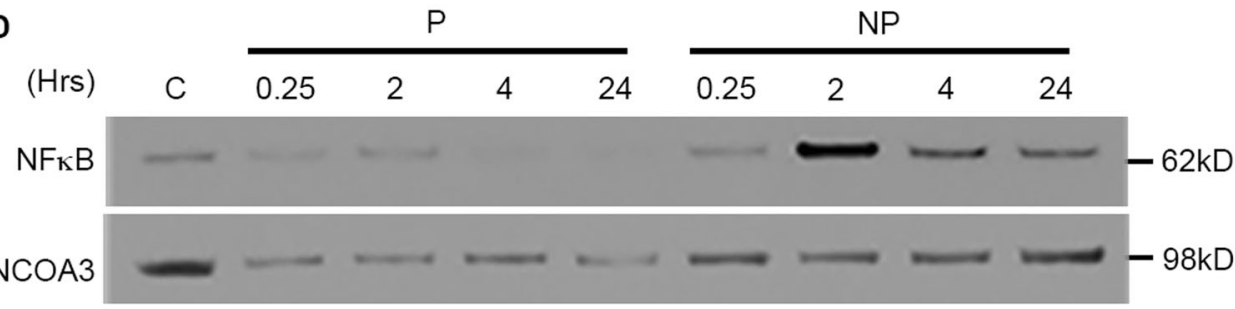

E

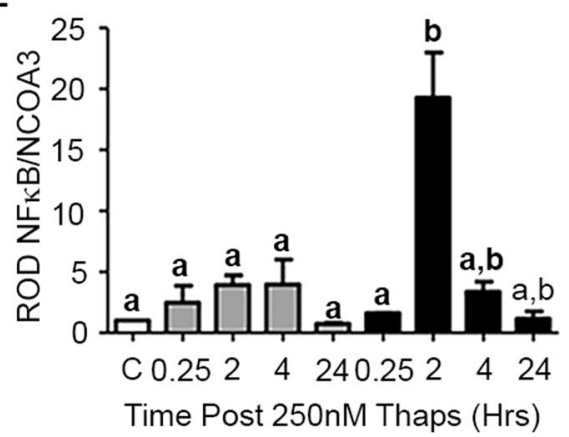

Fig. 2 UPR preconditioning ablates NF-KB activation in the hTERT-HM uterine myocyte. $\mathbf{a}, \mathbf{b}$, $\mathbf{d}$, e Activation of NF-KB was significantly increased in both TM and Thaps non-preconditioned (NP) cells and reduced to barely detectable levels in preconditioned (P) cells $2 \mathrm{~h}$ post administration of a cytotoxic dose of TM/Thaps. $\mathbf{c}$, $\mathbf{f}$ TNFa secretion was also reduced in P versus NP cells. A representative blot from each experiment is shown. NCOA3 is utilized as nuclear protein loading control. Statistical comparisons were performed using one-way ANOVA, and subsequent Newman-Keuls multiple-comparison tests. Data labeled with different letters are significantly different from each other $(p<0.05)$

$0.25,2,4$, and $24 \mathrm{~h}$ post administration of the cytotoxic bolus and compared to vehicle-treated controls. As seen in Fig. 2a, b, NP display a robust 5.5-fold activation of NF$\kappa \mathrm{B} 2 \mathrm{~h}$ post administration of the cytotoxic bolus, whereas NF- $\mathrm{kB}$ activation remains barely detectable in preconditioned cells at all time points examined post bolus $(0.25,2$, $4,24 \mathrm{~h})$.
Enzyme-linked immunosorbent assays (ELISA) performed on control preconditioned and NP hTERT-HM cells collected $48 \mathrm{~h}$ post TM bolus revealed TNF $\alpha$ secretion was suppressed 0.5 -fold in the preconditioned cells whereas, NP (Fig. 2c) demonstrated a 0.5-fold increase in levels compared to non-treated controls. Similar results were found when cells were preconditioned and stressed with Thaps (Fig. 2d). NF-kB activation and TNF $\alpha$ 

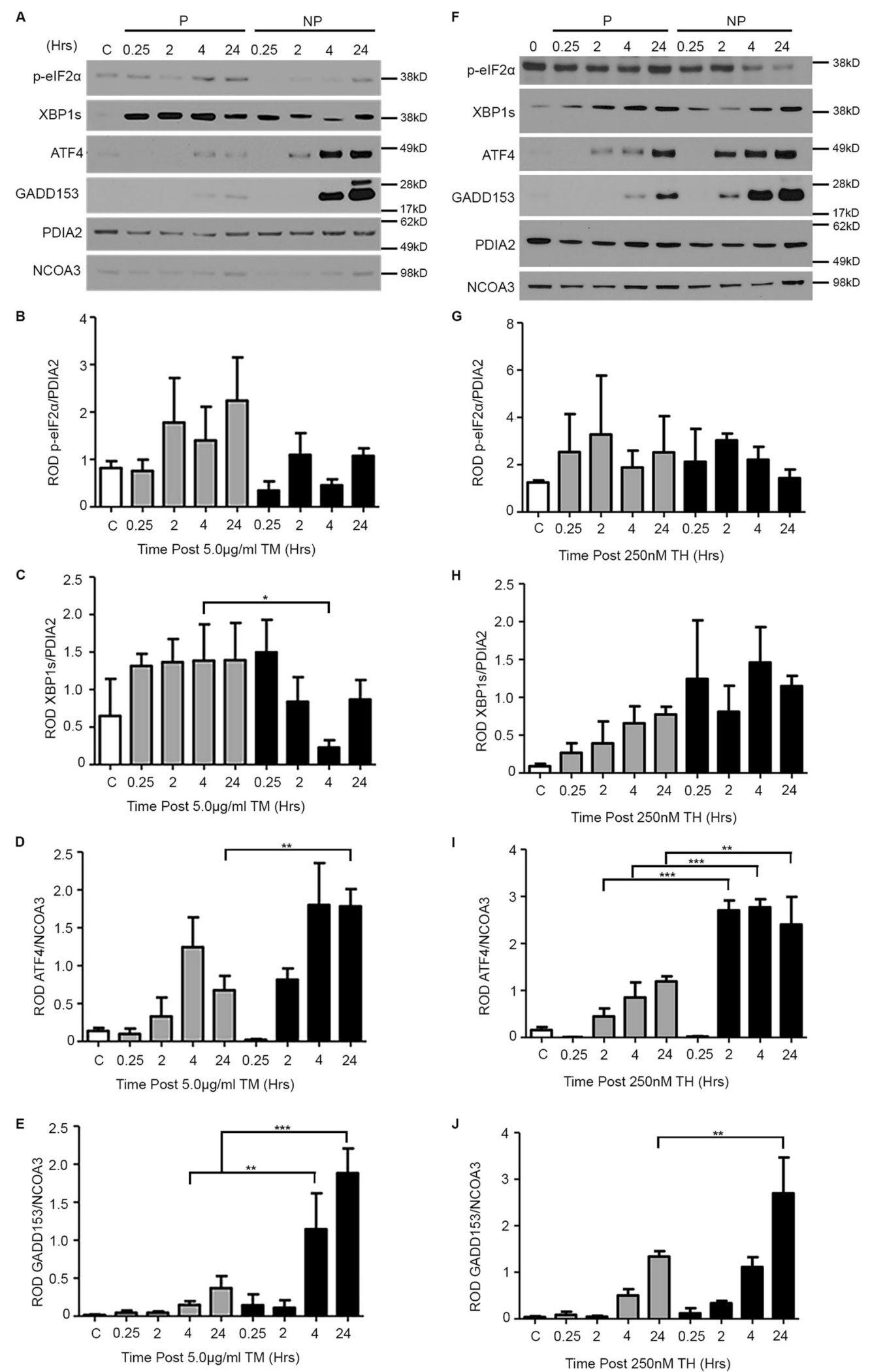

Fig. 3 (See legend on next page.) 
(see figure on previous page)

Fig. 3 UPR preconditioning differentially regulates activation of the pro and anti-apoptotic arms of the UPR in the hTERT-HM uterine myocyte. TM (a) or Thaps (f) mediated preconditioning blocked activation of the pro-apoptotic arms of the UPR with ATF4 (d) i) and GADD153 $(\mathbf{e}, \mathbf{j})$ and TM preconditioning maintained activation of the anti-apoptotic arm of the UPR with XBP1s (c) significantly upregulated in preconditioned (P) versus non-preconditioned (NP) cells post administration of a cytotoxic dose of TM/Thaps. No changes in XBP1s (h) upon Thaps treatment, and pelF2a (b, g) upon TM and Thaps treatment. PDIA2 and NCOA3 are utilized as cytoplasmic and nuclear protein loading controls. A representative blot from each experiment is shown. Statistical comparisons were performed using one-way ANOVA, and subsequent Newman-Keuls multiplecomparison tests. ${ }^{*} p<0.05,{ }^{* *} p<0.01$, and ${ }^{* *} p<0.001$ compared with controls

secretion was increased 19 -fold $2 \mathrm{~h}$ post and 4 -fold $48 \mathrm{~h}$ post exposure to the cytotoxic bolus (Fig. 2e, f) respectively, in NP but continued to remain suppressed in preconditioned cells.

\section{Preconditioning downregulates UPR activated apoptotic signaling}

We quantified stress-mediated activation of the UPR prosurvival (spliced XBP1 (XBP1s) and phosphoelongation initiation factor $2 \alpha$ (p-eIF $2 \alpha))$ and proapoptotic (ATF4 and GADD153 signaling pathways by immunoblotting immediately following the application of the TM or Thaps bolus $(0.25,2,4$, or $24 \mathrm{~h}$ post bolus) in control, preconditioned, and non-preconditioned hTERTHM cells (Fig. 3a, f). p-eIF2 $\alpha$ levels remain unchanged between preconditioned and NP, TM, or Thaps (Fig. 3b, g). XBP1s levels were suppressed $4 \mathrm{~h}$ post bolus in TMpreconditioned cells (Fig. 3c), whereas no change in expression is observed between Thaps-preconditioned and NP (Fig. 3h). Pro-apoptotic signaling pathways in contrast, were significantly downregulated in both TM and Thaps preconditioned versus NP. A 2-fold decrease in ATF4 at 24h (Fig. 3d) and a 7- and 5-fold reduction of GADD153 at 4 and $24 \mathrm{~h}$, respectively (Fig. 3e) was observed in TM-preconditioned cells. Similarly, a 0.5-fold reduction in ATF4 at $24 \mathrm{~h}$ (Fig. 3i) and a 2 -fold reduction in GADD153 at 2, 4, and $24 \mathrm{~h}$ (Fig. 3j) were observed in Thaps-preconditioned compared to non-preconditioned hTERT-HM cells.

Anti-apoptotic factors such as XIAP, MCL1, and Survivin have previously been demonstrated to increase in the uterine compartment of the pregnant mouse across gestation $^{19}$. Increased prosurvival signaling is thus thought to play a role in maintaining myometrial CASP3 in a non-apoptotic state throughout early and midgestation to prolong uterine quiescence. Thus we examined the effects of preconditioning on the expression of anti-apoptotic factors XIAP and MCL1. We observed that XIAP and MCL1 were preferentially maintained in TMpreconditioned cells (Supplementary Figure S4A, B and C). However, no significant differences were found in XIAP and MCL1 expression between Thaps-preconditioned, non-preconditioned, and control hTERT-HM cells (Supplementary Figure S4D, E and F).
Table 1 In vivo preconditioning prevents preterm birth

\begin{tabular}{llll}
\hline & Term birth & Preterm birth & Percent preterm \\
\hline Control & 3 & 0 & $0 \%$ \\
PBA & 3 & 0 & $0 \%$ \\
TM+PBA & 4 & 3 & $57 \%$ \\
TM & 1 & 5 & $17 \%$ \\
\hline
\end{tabular}

\section{Sub-preconditioned stressed mice have an increased incidence of preterm}

We examined the timing of labor following a minor stress $(0.2 \mathrm{mg} / \mathrm{kg} \mathrm{TM})$ on gestation day 16 in a subpreconditioned (TM + PBA) and an endogenously preconditioned (TM) population of timed pregnant mice ( $n=7$ and $n=6$, respectively). The effects of PBA and vehicle (Con) alone were also examined ( $n=3$ for both groups). About $57 \%$ of the TM + PBA, mice (4/7) delivered preterm, with an average delivery time of $30 \mathrm{~h}$ post TM administration (Table 1). In contrast, the mice that experienced normal endogenous gestational stressors prior to the delivery of a minor stress (TM) had a preterm birth rate of $17 \%$ (Table 1). No effects were observed in the timing of birth from the mice administered PBA alone $(3 / 3)$, similar to Con mice (3/3), which delivered at term on E19 (Table 1). All animals that delivered at term resulted in live pups.

We examined components of the inflammatory signaling cascade in the uteri collected from Con, PBA, TM + $\mathrm{PBA}$, and TM treated pregnant mice at E17 prior to the onset of term and preterm birth. Uterine NF- $\mathrm{kB}$ activation, macrophage infiltration, COX-1, and COX-2 levels were examined (Fig. 4). Premature uterine activation of NF- $\mathrm{KB}$ occurs in the stressed-sub-preconditioned (TM+PBA) mice prior to the onset of labor, a 2.7 -fold increase in p65 nuclear translocation was observed when compared to control animals (Fig. 4a). Immunohistochemistry validated the observed increased NF- $\mathrm{kB}$ activation in stressed-sub-preconditioned mice, and revealed the increased activity occurred within both the myometrial and endometrial compartments (Supplementary Figure S5A and B, respectively). While COX-2 levels remained undetectable, COX-1 levels significantly 

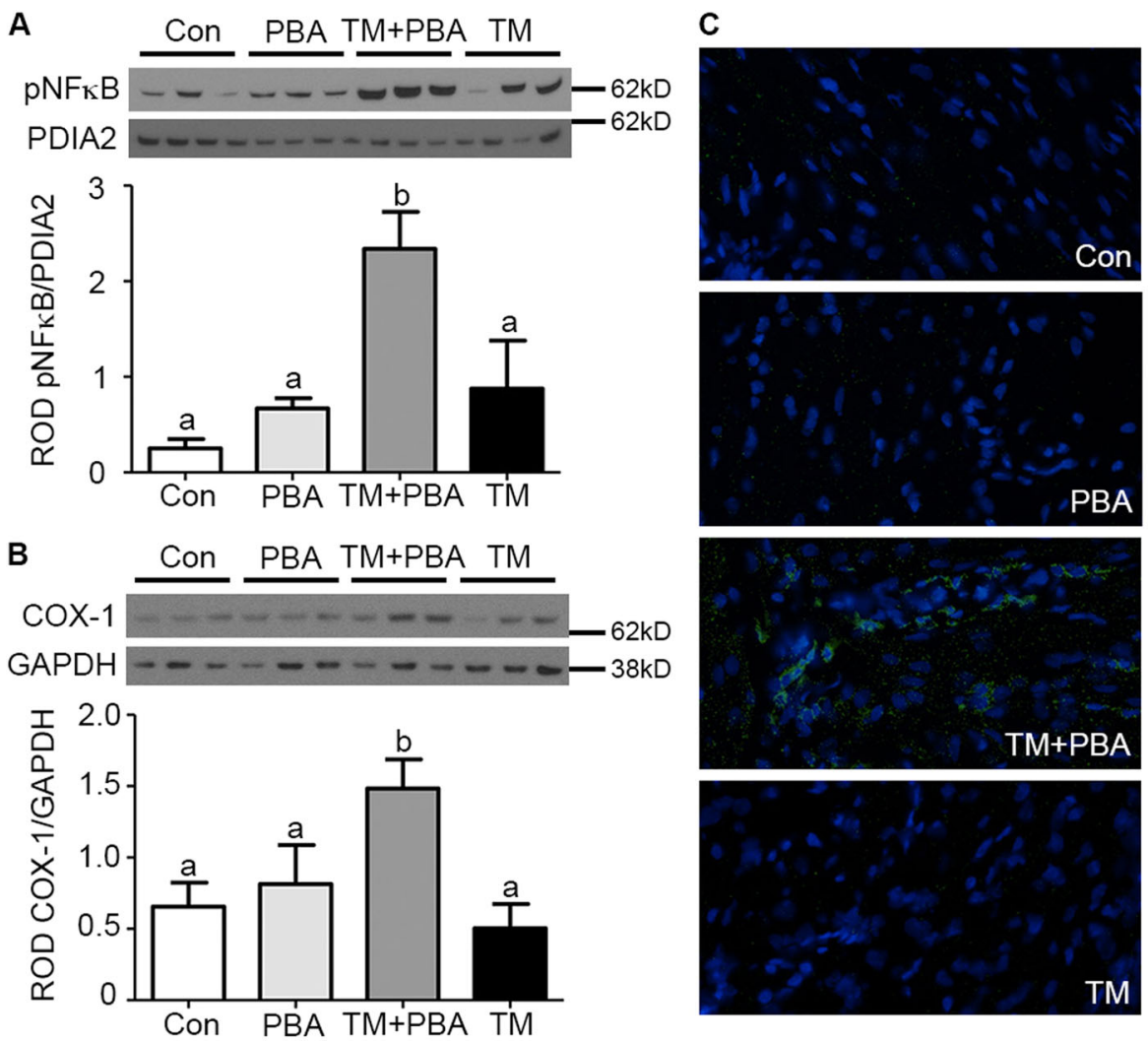

Fig. 4 Endogenous preconditioning prevents premature activation of uterine inflammation in the pregnant mouse. Uteri collected from vehicle treated (Con), sub-preconditioned (PBA), exogenously stressed-sub-preconditioned (TM+PBA) and exogenously stressed-preconditioned (TM) mice on E17 prior to the onset of preterm or term birth were examined for $\mathbf{a} \mathrm{NF}-\mathrm{KB}, \mathbf{b}$ COX-1, and $\mathbf{c}$ macrophage infiltration. Increased NF-KB activation, COX-1 expression, and elevated levels of macrophage infiltration were isolated to the TM+PBA uteri. PDIA2 and GAPDH are utilized as cytoplasmic loading controls. A representative blot or immunohistochemical image from each experiment is shown. Statistical comparisons were performed using one-way ANOVA, and subsequent Newman-Keuls multiple-comparison tests. Data labeled with different letters are significantly different from each other $(p<0.05)$

increased over 3-fold in the TM + PBA uteri (Fig. 4b) in comparison to Con uteri. Macrophage infiltration of the uterine tissue of the Con, PBA, TM+PBA and TM treated mice was examined by $\mathrm{F} 4 / 80+$ immunofluorescence analysis and a greater than 10 fold increase in the number of macrophages was observed in the TM + PBA uteri that consequently undergo preterm birth in comparison to the PBA, TM and Con uteri (Fig. 4c).

\section{Apoptotic CASP3 activity in the pregnant uterus increases contractility}

Uteri isolated from Con, PBA, TM + PBA, and TM mice were examined prior to the onset of term or preterm labor at E17 by immunoblotting for CASP3 activation. Levels of CASP3 activation were not significantly changed between the 4 groups examined (Fig. 5a). However, the $\mathrm{TM}+\mathrm{PBA}$ uteri demonstrate increased incidence of apoptotic CASP3 activation as indicated by a 4.6 -fold increase in the levels of uterine PARP cleavage (Fig. 5b) when compared to PBA, Con, or TM treated uteri.
Positive terminal deoxynucleotidyl transferase dUTP nicked-end labeling (TUNEL) staining in the subpreconditioned uteri, but not PBA, Con, or TM treated validated these results (Fig. 5c).

We observed a 2-fold decline in the homotetromeric non-active form of iPLA2 (Fig. 5d) in the apoptotic CASP3-positive (Fig. 5a, b) TM + PBA uteri in comparison to the Con and PBA-treated uteri. In the hTERT-HM we were able to detect the cleaved active form of iPLA2 and observed a 2-fold increase (Fig. 5e) isolated to the NP, which also display elevated levels of apoptotic CASP3 as indicated by the pattern of PARP cleavage in Fig. 1a.

Uteri isolated from Con, PBA, TM + PBA, and TM treated mice examined at E17 for prostaglandin production within the uterine compartment utilizing targetedsmall molecule liquid chromatography and tandem mass spectrometry. Significantly elevated levels of PGE2, PGE1, and PGD3 were isolated to the sub-preconditioned mice exposed to a minor exogenous stress (TM+PBA) (Fig. 6a, b, d). Furthermore, downstream by-products 

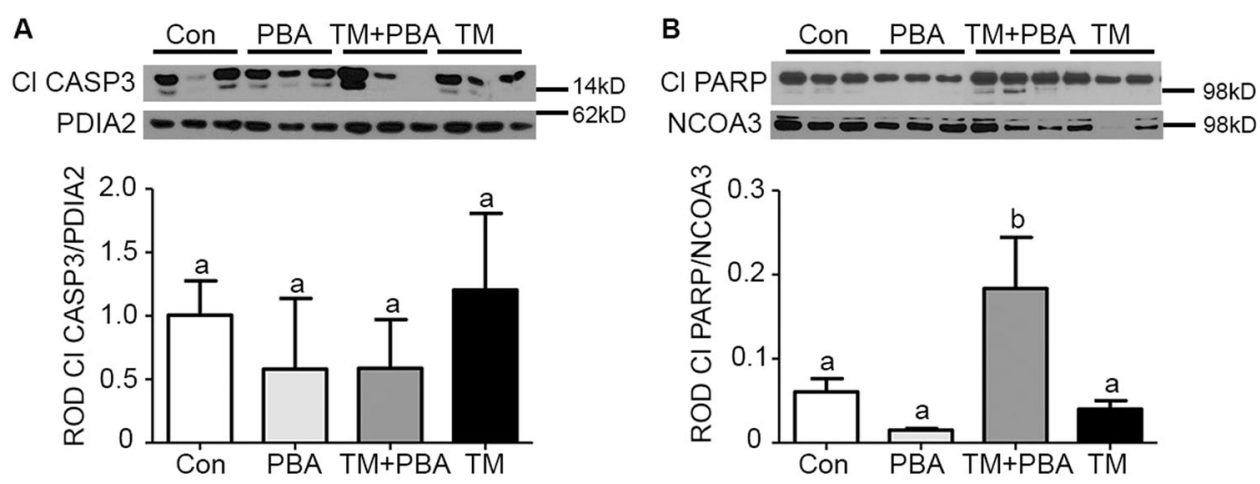

C
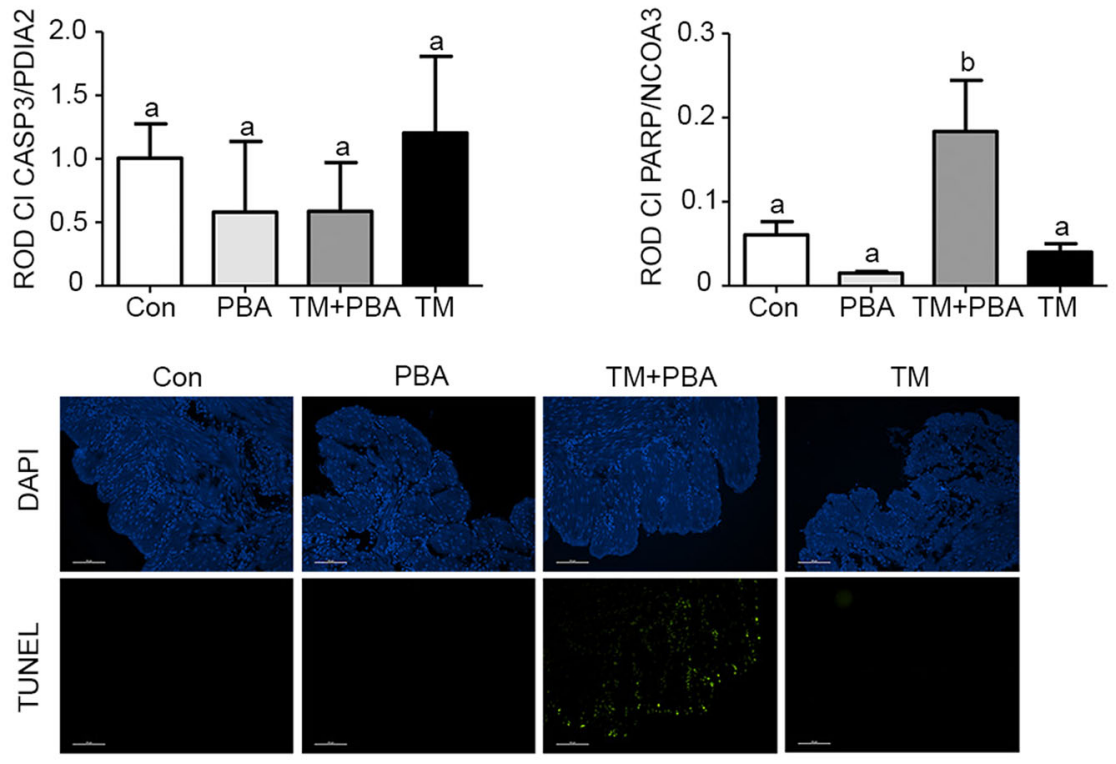

D

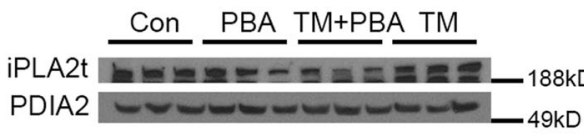

E
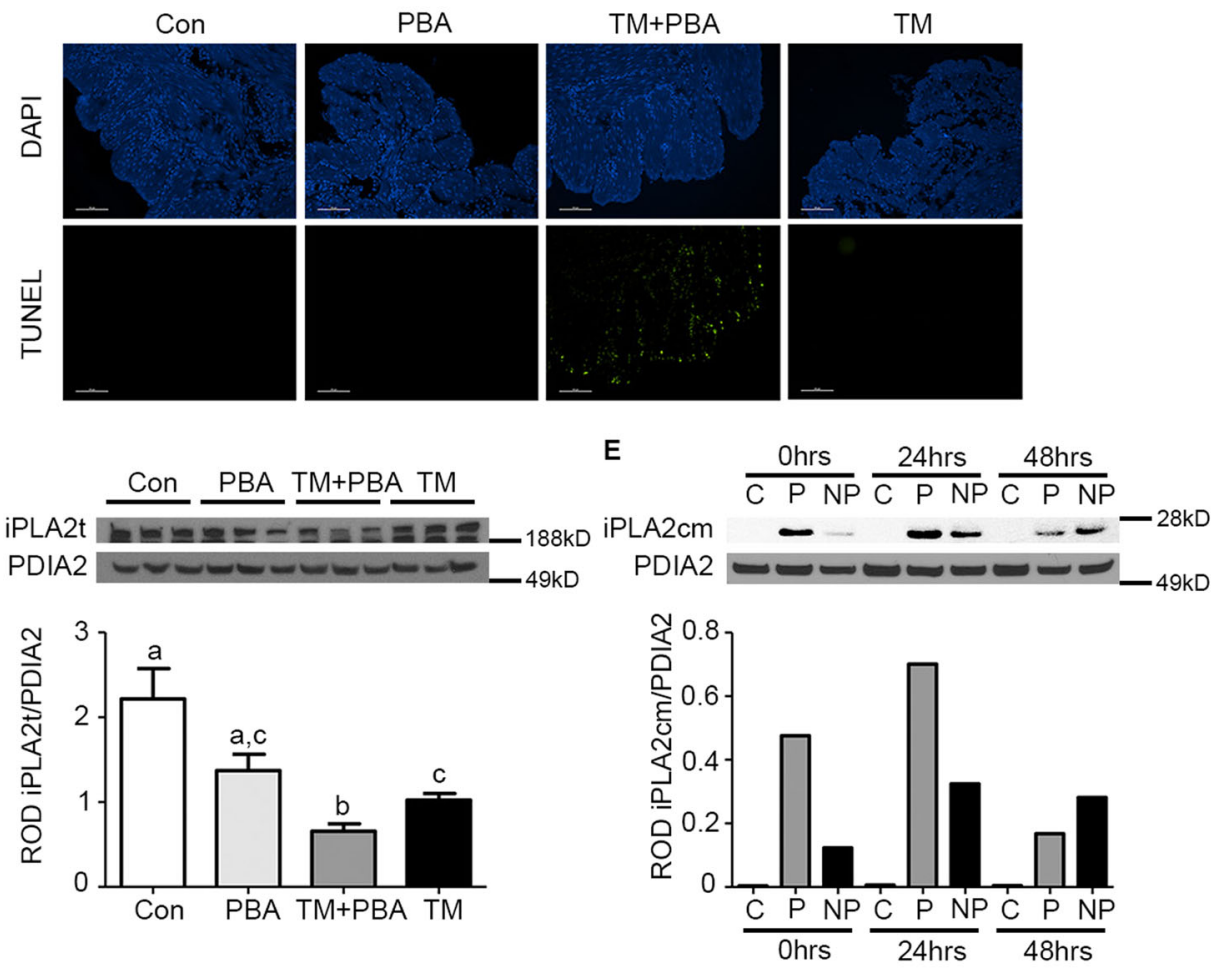

Fig. 5 Endogenous preconditioning facilitates the maintenance of non-apoptotic CASP3 and suppresses iPLA2 activation in the pregnant mouse uterus. Uteri collected from vehicle treated (Con), sub-preconditioned (PBA), exogenously stressed-sub-preconditioned (TM+PBA) and exogenously stressed-preconditioned (TM) mice on E17 prior to the onset of preterm and term birth were examined for a active CI CASP3. b CI PARP, and $\mathbf{c}$ TUNEL staining to measure apoptotic cell death. iPLA2t levels act as an indirect measure of iPLA2 activation (d). Cl CASP3 levels remained unchanged across all 4 groups examined however increased CI PARP and TUNEL activity and decreased levels of the inactive iPLA2t were isolated to the TM+PBA-treated mice. $\mathbf{e}$ In the hTERT-HM cells the cleaved active monomeric form of iPLA2 (iPLA2 cm) was elevated in a relative manner to the levels of apoptotic CASP3 present in the preconditioned and non-preconditioned cells (Fig. 1a). A representative blot or image from each experiment is shown. PDIA2 and NCOA3 are utilized as cytoplasmic and nuclear protein loading controls. Statistical comparisons were performed using one-way ANOVA, and subsequent Newman-Keuls multiple-comparison tests. Data labeled with different letters are significantly different from each other $(p<$ 0.05)

of arachidonic acid metabolism, e.g., 11-HETE and 13-HODE, were also significantly elevated in subpreconditioned mice compared to preconditioned controls (Supplementary Figure S6).

Ovaries were collected from Con, PBA, TM + PBA and TM treated pregnant mice on E17 and analyzed for $3 \beta$ hydroxysteroid dehydrogenase (HSD3B2), which declines with the onset of luteolysis. As seen in Fig. 6e, there was a significant reduction (2.2-fold) in HSD3B2 expression in the ovaries of the TM+PBA mice in comparison to Con, PBA, and TM ovaries. To validate premature luteolysis in $\mathrm{TM}+\mathrm{PBA}$ pregnant mice, serum collected on E17 from each cohort of mice was analyzed with ELISA for circulating progesterone levels. As observed in the TM+PBA 


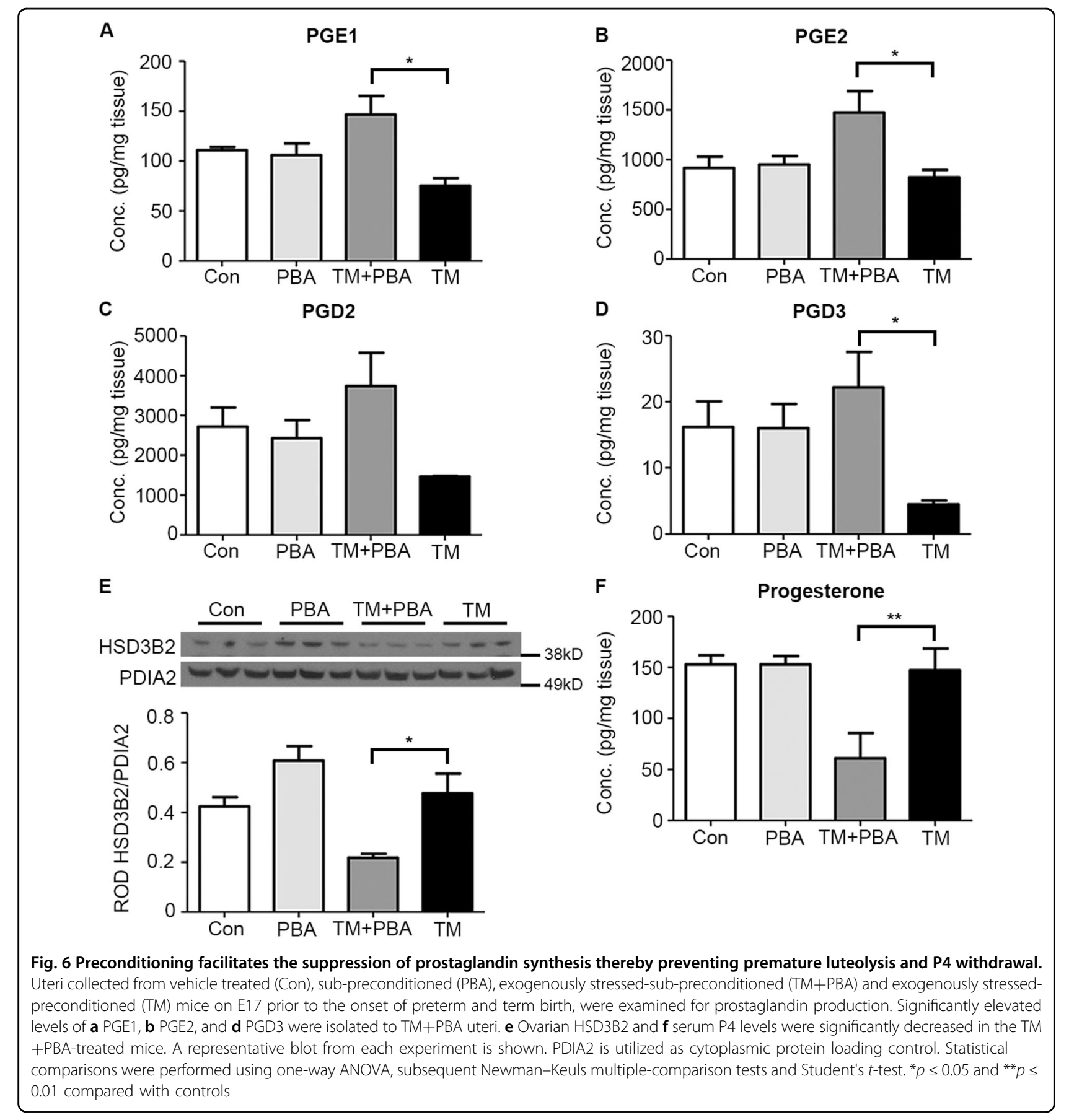

mice that undergo preterm delivery (Table 1), there was a significant decline (2.4-fold) in circulating P4 levels in comparison to Con, PBA, and TM treated mice (Fig. 6f).

\section{Discussion}

We have previously demonstrated the critical role the UPR plays in non-apoptotic CASP3 activation within the uterine compartment during pregnancy ${ }^{1}$. The current study is based on the findings that the act of preconditioning the uterine UPR during pregnancy is essential in protecting the pregnant uterine myocyte against a CASP3-mediated apoptotic fate. We also demonstrate that uterine preconditioning of the UPR during pregnancy promotes prolonged uterine myocyte quiescence through the suppression of apoptotic CASP3mediated activation of two interdependent signaling pathways, the inflammatory and iPLA2-prostaglandin signaling cascade. Increased uterine inflammation and 
prostaglandin signaling herald the end of gestation and are normally associated with the onset of labor in both human and mouse ${ }^{20}$. Consequently, we propose that in the absence of appropriate preconditioning or an inability to host an appropriate UPR to preconditioning stimuli, the uterine compartment is placed at an increased risk for precocious apoptotic CASP3 activation, which results in a heightened incidence of preterm birth.

CASP3 activity is commonly associated with the initiation of cellular apoptosis ${ }^{21}$. However it has repeatedly been demonstrated that despite high levels of active CASP3, the pregnant myometrium avoids cellular apoptosis throughout the entirety of gestation and postpartum involution, whereas the endometrium displays increased apoptotic CASP3 activity at term and during the involution process ${ }^{22,23}$. While CASP3 targets were not identified in the smooth muscle of the bladder, CASP3 was found to target structure proteins in both the heart and diaphragm. In cardiac tissue specifically, both $\alpha$-actin and $\alpha$-actinin, components of the cardiac contractile architecture were directly cleaved by CASP3 and then further degraded ${ }^{13-}$ 15. Similarly, we have shown in the absence of apoptotic consequences non-apoptotic uterine CASP3 protease activity inhibits myometrial contraction in a tocolytic manner through the targeted cleavage and degradation of multiple components of the contractile architecture, i.e., connexin $43, \alpha$-actin, and $\gamma$-actin, limiting uterine myocyte contractile potential ${ }^{1,19}$.

Preconditioning refers to the phenomenon where sublethal insults can induce tolerance to subsequent stressors. The process of preconditioning biological systems against pathophysiological events can be observed in multiple forms. As an example, ischemia/reperfusion preconditioning has become an increasingly active area of interes $^{24-26}$ and it has been demonstrated that multiple applications of brief ischemic events prior to prolonged ischemia significantly reduces subsequent tissue damage and limits the apoptotic outcomes associated with CASP3 activation $^{3-5}$. Both in vivo and in vitro studies have proven that preconditioning acts through the endoplasmic reticulum with minor insults of stress stimulating a protective adaptive UPR promoting tolerance and antiapoptotic signaling fostering resistance to the apoptotic processes associated with subsequent, more damaging stresses $^{18,27}$. This preconditioning phenomenon is strongly conserved in evolution ${ }^{28}$ and can be induced by many and varied perturbations such as stress-mediated inflammation, hypoxia, and cytotoxins ${ }^{18,29,30}$. Prior to this study, data pertaining to the role of preconditioning the uterine UPR in the regulation of gestational length was limited. It was known however, that women who exercise during pregnancy have a reduced risk of preterm delivery $^{31}$. Whereas exercise in non-pregnant women has been demonstrated to acutely activate the UPR to facilitate adaptation of skeletal muscle ${ }^{32}$. Exercising while pregnant may be acting in a preconditioning-like manner to increase myometrial tolerance to subsequent stressors. More relevantly, studies in nonhuman primates examining the effects of uterine overdistention on preterm labor have found significant evidence of uterine preconditioning $^{33}$. Specifically, they show that additional stretch to the pregnant uterus at approximately the start of the third trimester via intraamniotic balloons lead to the onset of preterm labor. Yet, when the days to maximum inflation of the balloons were extended from 1 day to 2 days, preterm labor did not occur and all nonhuman primates delivered at term. Importantly, the maximum volume or stretch induced did not differ between the two experiments. While these studies did not delve into the mechanism of sustained quiescence they do suggest the initial stretch stimulus to the pregnant uterus facilitated myometrial adaption preventing preterm labor in response to the subsequent stretch. Our in vitro data reveal preconditioning of the uterine myocyte UPR, suppressed inflammatory responses (Fig. 2), enhanced prosurvival signaling (Fig. 3), and promoted non-apoptotic CASP3 activation (Fig. 1) during periods of cellular stress. As can be observed in Fig. 1, despite equally elevated levels of CASP3 activation (Fig. 1c) in preconditioned and NP $48 \mathrm{~h}$ post receiving a bolus (Fig. 1), the preconditioned cells demonstrated a newly acquired resistance to CASP3mediated apoptotic cell death as indicated by decreased PARP cleavage (Fig. 1d). Suppressed activation of NF-kB and consequently decreased secretion of TNFa (Fig. 2c) were also observed upon uterine myocyte UPR preconditioning (Fig. 2). Furthermore preconditioning of the UPR promotes inhibition of the pro-apoptotic arms and enhanced activation of the protective arms of the UPR, respectively (Fig. 3, Supplementary Figure S1). Taken together these data demonstrate that preconditioning of the uterine myocyte plays a critical role in maintaining the uterine myocyte in a CASP3-positive, non-apoptotic, antiinflammatory, prosurvival phenotype in vitro. Our in vivo analysis of the stressed-preconditioned and stressed-subpreconditioned pregnant mouse uteri established that the preconditioned non-apoptotic phenotype is critical for the maintenance of an appropriate gestational length through the preservation of uterine quiescence. In vivo analysis of a stressed-sub-preconditioned pregnant mouse model (TM+PBA) revealed they were more susceptible to a preterm birth phenotype (57\%) than mice that experienced normal endogenous preconditioning prior to the delivery of a minor exogenous stress (TM), which displayed a preterm birth rate of only $17 \%$ (Table 1 ). As can be observed in Fig. 4a, the uterus of the TM+PBA mice also demonstrated significantly elevated levels of macrophage infiltration, NF- $\mathrm{\kappa B}$ activation, and COX-1 expression (Fig. 4a-c). These data demonstrate that appropriate 
endogenous preconditioning maintains the uterine capacity to suppress inflammatory signaling cascades late in gestation within the uterine compartment protecting against subsequent exogenous stressors. Moreover, while uterine CASP3 levels remained unmodified across treatments (Fig. 5a), the stressed-sub-preconditioned mice (TM+PBA) displayed increased uterine apoptotic CASP3 as indicated by both increased PARP cleavage (Fig. 5b) and elevated TUNEL staining observed primarily within the endometrial compartment (Fig. 5c). These data demonstrate that preconditioning plays a vital role in protecting the endogenously stressed uterine compartment from undergoing a precocious apoptotic CASP3mediated cell death.

It has previously been established that CASP3 activity is a critical upstream component in prostaglandin production through cleavage and activation of iPLA2 allowing the release of free arachidonic acid to be converted into prostaglandins in a COX1/2- and NF-kB-dependent manner $^{34,35}$. However, this study reveals that it must be apoptotic CASP3 activity for the appropriate initiation of prostaglandin synthesis while non-apoptotic CASP3 though active is unable to do so. Upon apoptotic CASP3 activation (TM+PBA), we observed decreased levels of the inactive uterine iPLA2 in vivo (Fig. $5 \mathrm{c}, \mathrm{d}$ ). In vitro the cleaved active form of iPLA2 was readily detectable (Fig. 5e) and found to be significantly upregulated in the presence of apoptotic CASP3 and reduced in the presence of non-apoptotic CASP3 (48 h P versus NP Fig. 1a). iPLA2 activation in response in precocious apoptotic CASP3 in the uterine compartment of the TM+PBA mouse resulted in precocious prostaglandin production (Fig. 6), triggering luteolysis as evidenced by decreased ovarian HSD3B2 (Fig. 6e ${ }^{36}$ and consequently decreased circulating P4 levels (Fig. $6 \mathrm{f}$ ) and the onset of preterm birth limited to the TM+PBA-treated mice (Table 1).

Taken together, these data demonstrate for the first time that uterine preconditioning, acting to suppress uterine apoptotic CASP3 activation and inflammation is placed upstream of regulating the timing of normal parturition by actively preventing the well-established endogenous uterotonic signaling cascades such as prostaglandin synthesis, luteolysis, and consequently $\mathrm{P} 4$ withdrawal that herald the onset of normal term labor in the pregnant mouse.

\section{Materials and methods}

\section{Animals}

The Institutional Animal Care and Use Committee of Wayne State University approved all animal studies. Timed pregnant female CD-1 mice (6-8 wks; gestation day 9) (Charles River Laboratories, Wilmington, MA) were housed in AALAC-accredited facilities according to IACUC guidelines. Accordingly, mice were given a standard pellet diet and water ad libitum.

\section{Cell culture}

For the in vitro cell culture model system, we utilized telomerase immortalized human myometrial cells (hTERT-HM). ${ }^{17}$ In detail, human myometrial cells were collected from the anterior wall of the uterine fundus in women of reproductive age undergoing a hysterectomy. The catalytic subunit of telomerase was then expressed in the myometrial cells via retroviral infection. In these studies, hTERT-HM cells were cultured in Dulbeccomodified Eagle/F12 low glucose media (DMEM-F12) (Invitrogen Carlsbad, CA), supplemented with 10\% fetal bovine serum (vol/vol) (Invitrogen) and antibiotic/antimycotic (10,000 U/ml; Invitrogen), and incubated at $37^{\circ} \mathrm{C}$ with $95 \%$ air and $5 \% \mathrm{CO}_{2}$.

\section{TM and thaps treatments in vitro}

TM was suspended in $20 \mu \mathrm{l} 10 \mathrm{M}$ sodium hydroxide and brought to a final concentration of either $0.1 \mu \mathrm{g} / \mathrm{ml}$ or $1.0 \mu \mathrm{g} / \mathrm{ml}$ in DMEM-12 media with $10 \%$ FBS and antibiotic/antimycotic. Thaps (Sigma-Aldrich, St. Louis, MO; Cat\#T9033) was dissolved directly in cell culture media and brought to a final concentration of $10 \mathrm{nM}$ or $250 \mathrm{nM}$. For TM preconditioned (P) and non-preconditioned (NP) treatments hTERT-HM were given a $24 \mathrm{~h}$ treatment of $0.1 \mu \mathrm{g} / \mathrm{ml} \mathrm{TM}$ or vehicle, respectively, $0-48 \mathrm{~h}$ prior to a secondary treatment of $5.0 \mu \mathrm{g} / \mathrm{ml}$ TM. Similarly, for Thaps preconditioned (P) and non-preconditioned (NP) treatments hTERT-HM cells were given a $24 \mathrm{~h}$ treatment of $10 \mathrm{nM} \mathrm{TH}$ or vehicle, respectively, $48 \mathrm{~h}$ prior to a secondary treatment of $250 \mathrm{nM}$ Thaps. In both conditions, media were replaced $1 \mathrm{~h}$ after the secondary treatment was given and cells and media were collected $47 \mathrm{~h}$ later.

\section{TM and PBA treatments in vivo}

PBA was directly dissolved into phosphate buffered saline (PBS) at pH 8.0 (Santa Cruz Biotechnology, Dallas, TX; sc-200652). TM (Calbiochem, San Diego, CA; Cat\#654380) was initially dissolved in $20 \mu \mathrm{l} 10 \mathrm{M}$ sodium hydroxide and then suspended in PBS, pH 8.0. Subpreconditioned pregnant CD-1 female mice (E10-15) were administered twice-daily intraperitoneal injections (i.p) of $50 \mathrm{mg} / \mathrm{kg} \mathrm{PBA}$, while preconditioned controls were administered PBS. At E16, stressed mice were administered $0.2 \mathrm{mg} / \mathrm{kg}$ TM i.p, while controls were given volume matched PBS. Following TM injections, the length of gestation was then monitored and compared between a subset of sub-preconditioned and endogenously preconditioned mice. Uteri, ovaries, and serum were harvested at $\mathrm{E} 17$ in the additional mice.

\section{Cytosol and nuclear protein fractionation}

Cytoplasmic and nuclear protein fractions from hTERT-HM cells and frozen mouse tissues were prepared 
as previously mentioned. Initially, cells were rinsed in icecold PBS, centrifuged at $956 \times g$, re-suspended, and evenly homogenized in ice-cold NE1 buffer $(10 \mathrm{mM}$ Hepes $\mathrm{pH}$ $7.5,10 \mathrm{mM} \mathrm{MgCl}_{2}, 5 \mathrm{mM} \mathrm{KCl}, 0.1 \%$ Triton X-100 with $1 \mathrm{X}$ EDTA-free protease/phosphatase inhibitor mini tablet). While tissues, were pulverized in liquid nitrogen and homogenized in ice-cold NE1 buffer. The homogenates were then centrifuged at $2655 \times g$, the supernatant was retained as the cytoplasmic protein fraction and the pellet was washed in NE1 buffer and suspended in ice-cold NE2 buffer $(20 \mathrm{mM}$ Hepes $\mathrm{pH} 7.9,500 \mathrm{mM} \mathrm{NaCl}, 1.5 \mathrm{mM}$ $\mathrm{MgCl}_{2}, 0.2 \mathrm{mM}$ EDTA pH 8.0, 25\% (vol/vol) glycerol with 1X EDTA-free protease/phosphatase inhibitor mini tablet). The homogenate was vortexed for $30 \mathrm{~s}$ every $5 \mathrm{~min}$ and after $1 \mathrm{~h}$, centrifuged at $10,621 \times g$. The supernatant was then retained as the nuclear fraction. Protein estimation was performed using a bicinchoninic acid (BCA) assay, equal amounts of protein were loaded for immunoblotting, PDI and NCOA3 were utilized as loading controls for the cytoplasmic and nuclear fractions, respectively.

\section{Immunoblotting and densitometric analysis}

Equal amounts of protein were separated via electrophoresis on NuPAGE 4-12\% gradient precast polyacrylamide gels (Life Technologies, Carlsbad, CA). Proteins were transferred onto Hybond-P PVDF membranes (Millipore, Billerica, MA) and blocked for $1 \mathrm{~h}$ at room temperature in $5 \%$ non-fat milk prepared in Tris Buffered Saline with 0.1\%Tween-20 (vol/vol). Membranes were incubated with primary antibodies overnight at $4{ }^{\circ} \mathrm{C}$. Primary antibody concentrations were as follows: GRP78 (1:1000; Cat\#3177), Cl CASP3 (1:250; Cat\#9664), GADD153 (1:500; Cat\#5554), Cl PARP (1:1000; Cat\#9541), ATF4 (1:500; Cat\#11815), p-eIF2 $\alpha$ (1:500; Cat\#3398), NF-kB (1:1000; Cat\#8242), XIAP (1:250; Cat\#2042), and PDI (1:5000; Cat\#3501) were obtained from Cell Signaling Technologies; XBP1s (1:500; Cat\#37152) was obtained from Abcam; ATF6 (1:500; Cat\#24169-1-AP) was obtained from Proteintech; MCL1 (1:1000; Cat\#sc-819) was obtained from Santa Cruz Biotechnology; and NCOA3 (1:5000; Cat\#PA1-845) was obtained from ThermoScientific. Following primary incubation, immunoreactivity was detected using horseradish peroxidase-conjugated secondary antibodies and visualized using an enhanced-chemiluminescence detection system (ThermoScientific, Rockford, IL). Immunoreactive band density was then quantified using ImageJ software.

\section{Enzyme-linked immunosorbent assay (ELISA)}

In vitro, media samples were loaded into Amicon Ultra Centrifugal Filters (Millipore, cat\#UFC500396) and centrifuged for $30 \mathrm{~min}$ at $14,000 \times g$ to concentrate media $\sim 10 \times$. The level of human tumor necrosis factor-alpha (TNF $\alpha$ ) was then measured in 10X concentrated media using an ELISA. Specifically, the MSD Multi-Spot TNFa ELISA (Meso Scale Diagnostics, Rockville, MD, Cat\#K151QWD) was performed according to the manufacturer's instructions and results were read via the Meso Scale Discovery 1300 microplate reader. Each sample measurement was read in duplicate and the computed averages were taken based on the calculated standard curve.

In vivo, the level of progesterone (P4) was then measured in pregnant mouse serum using an ELISA. Specifically, the P4 ELISA Kit (Alpha Diagnostic International, San Antonio, TX, Cat\#1955) was performed according to the manufacturer's instructions and results were read via the Molecular Devices, SpectraMax M2 microplate reader. Each sample measurement was read in duplicate and the computed averages were taken based on the calculated standard curve.

\section{Terminal deoxynucleotidyl transferase dUTP nicked-end labeling assay}

Tissues collected at E17, imbedded in optimal cutting temperature compound (Sakura Finetek USA Inc, Torrance, $C A$ ) were sectioned (10- $\mu \mathrm{m}$ thick), mounted onto Superfrost Plus Micro Slides, and stored at $-20^{\circ} \mathrm{C}$. Sections were removed from storage and fixed in $4 \%$ paraformaldehyde for $15 \mathrm{~min}$. Additionally, sectioned paraffin wax imbedded tissues were de-paraffinized and rehydrated and treated with $10 \mu \mathrm{g} / \mathrm{ml}$ Proteinase $\mathrm{K}$ for $15 \mathrm{~min}$ at $37^{\circ} \mathrm{C}$. Analysis of apoptosis in all tissues was quantified using the In Situ Cell Death Detection Kit, AP (Roche, Indianapolis, IN, Cat\#11684809910) according to the manufacturer's instructions.

\section{Small molecule liquid chromatography mass spectrometry analysis}

Dissected uterine tissues separated into endometrial and myometrial compartments, flash frozen in liquid nitrogen, were removed from $-80^{\circ} \mathrm{C}$ storage and tissue weights were immediately recorded. Samples were then suspended in $1 \mathrm{ml}$ cold $\mathrm{PBS} \mathrm{pH}$ 7.4, homogenized via bead homogenization, and centrifuged at $10,621 \times g$ for $10 \mathrm{~min}$. Supernatants were removed, and protein concentrations were determined using a BCA assay. Equal volume of protein $(850 \mu \mathrm{l})$ was then spiked with $5 \mathrm{ng}$ of internal standards suspended in $15 \%$ methanol dissolved in water $(150 \mu \mathrm{l})$, mixed thoroughly, and purified using a C18 solid-phase cartridges. Prior to applying the sample, the cartridges were first washed with $1 \mathrm{ml}$ of $100 \%$ methanol followed by $1 \mathrm{ml}$ of $15 \%$ methanol. After the addition of the sample, tubes were rinsed twice with $1 \mathrm{ml}$ of PBS and the rinse was passed through the cartridges. Subsequently, the cartridges were rinsed with $2 \mathrm{ml}$ of hexane, vacuum 
dried for $30 \mathrm{~s}$ and proteins were eluted with $1 \mathrm{ml}$ of methanol containing $0.1 \%$ formic acid. All samples were evaporated to dryness with a gentle stream of nitrogen at $40^{\circ} \mathrm{C}$, residues were re-suspended in $30 \mu \mathrm{l}$ methanol and stored at $-20^{\circ} \mathrm{C}$ until LC-MS analysis. Prior to analysis, each sample was further diluted with $30 \mu \mathrm{l} 25 \mathrm{mM}$ aqueous ammonium acetate. Specific methods utilized for liquid chromatography mass spectrometry can be referenced in Yoon Park et al. 2014.

\section{Immunofluorescence}

Tissues collected at E17, embedded in optimal cutting temperature compound (OCT) (Sakura Finetek USA Inc, Torrance, CA) were sectioned $(10 \mu \mathrm{m}$ thick), mounted onto Superfrost Plus Micro Slides, and stored at $-20{ }^{\circ} \mathrm{C}$. Sections removed from storage were fixed in $4 \%$ paraformaldehyde for $2 \mathrm{~min}$. Fixed sections were incubated with primary antibody overnight at $4{ }^{\circ} \mathrm{C}$ and examined for primary immunoreactivity using a conjugated secondary antibody. The primary and secondary antibody concentrations were as follows: F4/80+(1:250, Abcam, Cat\#ab6640) diluted in PBS and detected by secondary goat anti-rat antibody conjugated to Alexa Fluor 488 (1:500, Abcam, Cat\#150157), NF-kB (1:400, Cell Signaling Technologies, Cat\#8242) diluted in PBS and detected by secondary donkey anti-rabbit antibody conjugated to Cy3 (1:500, Jackson Immunoresearch, Cat\#711-165-152).

\section{Statistical analysis}

All data represent at least three individual experiments performed in triplicate. For the direct comparison of three or more conditions a one-way analysis of variance was performed, with multiple comparisons analyzed via Newmans-Keuls multiple comparisons test. When directly comparing two conditions a two-tailed Student's $t$-test was performed. All comparisons were considered significant with $p$-values less than 0.05 .

\section{Funding}

This work was supported by the March of Dimes \#21 FY2012-152 and the NICHD 1R01HD06511

\author{
Author details \\ ${ }^{1}$ Department of Physiology, School of Medicine, Wayne State University, \\ Detroit, MI, USA. ²Department of Biology, University of Detroit Mercy, Detroit, \\ MI, USA. ${ }^{3}$ Department of Obstetrics and Gynecology, Wayne State University, \\ Detroit, MI, USA. ${ }^{4}$ Perinatal Research Initiative in support of the Perinatology \\ Research Branch, Division of Obstetrics and Maternal-Fetal Medicine, Division \\ of Intramural Research, Eunice Kennedy Shriver National Institute of Child \\ Health and Human Development, National Institutes of Health, U. S. \\ Department of Health and Human Services, Bethesda and Detroit, MD and MI, \\ USA

\section{Conflict of interest} \\ The authors declare that they have no conflict of interest.
}

\section{Publisher's note}

Springer Nature remains neutral with regard to jurisdictional claims in published maps and institutional affiliations.

Supplementary Information accompanies this paper at (https://doi.org/ 10.1038/s41419-018-1000-4).

Received: 3 April 2018 Revised: 24 July 2018 Accepted: 27 July 2018 Published online: 17 September 2018

\section{References}

1. Kyathanahalli, C. et al. Uterine endoplasmic reticulum stress-unfolded protein response regulation of gestational length is caspase-3 and -7-dependent. Proc. Natl Acad. Sci. USA 112, 14090-14095 (2015).

2. Jeyasuria, P., Wetzel, J., Bradley, M., Subedi, K. \& Condon, J. C. Progesteroneregulated caspase 3 action in the mouse may play a role in uterine quiescence during pregnancy through fragmentation of uterine myocyte contractile proteins. Biol. Reprod. 80, 928-934 (2009).

3. McLaughlin, B. et al. Caspase 3 activation is essential for neuroprotection in preconditioning. Proc. Natl Acad. Sci. USA 100, 715-720 (2003).

4. McLaughlin, B. The kinder side of killer proteases: caspase activation contributes to neuroprotection and CNS remodeling. Apoptosis 9, 111-121 (2004).

5. Tanaka, $H$. et al. Ischemic preconditioning: neuronal survival in the face of caspase-3 activation. J. Neurosci. 24, 2750-2759 (2004).

6. Shynlova, O. et al. Myometrial apoptosis: activation of the caspase cascade in the pregnant rat myometrium at midgestation. Biol. Reprod. 74, 839-849 (2006).

7. Shynlova, O., Kwong, R. \& Lye, S. J. Mechanical stretch regulates hypertrophic phenotype of the myometrium during pregnancy. Reproduction 139, 247-253 (2010).

8. Shynlova, O., Tsui, P., Jaffer, S. \& Lye, S. J. Integration of endocrine and mechanical signals in the regulation of myometrial functions during pregnancy and labour. Eur. J. Obstet. Gynecol. Reprod. Biol. 144, S2-S10 (2009).

9. Puri, C. P. \& Garfield, R. E. Changes in hormone levels and gap junctions in the rat uterus during pregnancy and parturition. Biol. Reprod. 27, 967-975 (1982).

10. Gomez-Lopez, N., StLouis, D., Lehr, M. A., Sanchez-Rodriguez, E. N. \& ArenasHernandez, M. Immune cells in term and preterm labor. Cell Mol. Immunol. 11, 571-581 (2014)

11. Sokolowski, P. et al. Human uterine wall tension trajectories and the onset of parturition. PLOS ONE 5, e11037 (2010).

12. Suresh, A., Subedi, K., Kyathanahalli, C., Jeyasuria, P. \& Condon, J. C. Uterine endoplasmic reticulum stress and its unfolded protein response may regulate caspase 3 activation in the pregnant mouse uterus. PLOS ONE 8, e75152 (2013).

13. Hong, S. K., Son, H., Kim, S. W., Oh, S. J. \& Choi, H. Effect of glycine on recovery of bladder smooth muscle contractility after acute urinary retention in rats. BJU Int 96, 1403-1408 (2005).

14. Communal, C. et al. Functional consequences of caspase activation in cardiac myocytes. Proc. Natl Acad. Sci. USA 99, 6252-6256 (2002).

15. Supinski, G. S. \& Callahan, L. A. Caspase activation contributes to endotoxininduced diaphragm weakness. J. Appl. Physiol. 100, 1770-1777 (2006).

16. Ingles, J., Kyathanahalli, C., Jeyasuria, P. \& Condon, J. C. Thinking outside the box. J. Cardiovasc Pharmacol. Ther. 22, 337-346 (2017).

17. Condon, J. C. et al. Telomerase immortalization of human myometrial cells. Biol. Reprod. 67, 506-514 (2002).

18. Peyrou, M. \& Cribb, A. E. Effect of endoplasmic reticulum stress preconditioning on cytotoxicity of clinically relevant nephrotoxins in renal cell lines. Toxicol. Vitr. 21, 878-886 (2007).

19. Jeyasuria, P., Subedi, K., Suresh, A. \& Condon, J. C. Elevated levels of uterine anti-apoptotic signaling may activate NFKB and potentially confer resistance to caspase 3-mediated apoptotic cell death during pregnancy in mice. Biol. Reprod. 85, 417-424 (2011).

20. Ratajczak, C. K. \& Muglia, L. J. Insights into parturition biology from genetically altered mice. Pediatr. Res 64, 581-589 (2008).

21. Lazebnik, Y. A., Kaufmann, S. H., Desnoyers, S., Poirier, G. G. \& Earnshaw, W. C. Cleavage of poly(Adp-Ribose) polymerase by a proteinase with properties like ice. Nature 371, 346-347 (1994). 
22. Kyathanahalli, C. et al. Cross-species withdrawal of MCL1 facilitates postpartum uterine involution in both the mouse and baboon. Endocrinology 154, 4873-4884 (2013).

23. Stephenson-Famy, A. et al. Antiapoptotic signaling via MCL1 confers resistance to caspase-3-mediated apoptotic cell death in the pregnant human uterine myocyte. Mol. Endocrinol. 26, 320-330 (2012).

24. Kume, M. et al. Ischemic preconditioning of the liver in rats: implications of heat shock protein induction to increase tolerance of ischemia-reperfusion injury. J. Lab. Clin. Med. 128, 251-258 (1996).

25. Przyklenk, K., Bauer, B., Ovize, M., Kloner, R. A. \& Whittaker, P. Regional ischemic 'preconditioning' protects remote virgin myocardium from subsequent sustained coronary occlusion. Circulation 87, 893-899 (1993).

26. Garcia, L. et al. Ischaemic preconditioning in the rat brain: effect on the activity of several initiation factors, Akt and extracellular signal-regulated protein kinase phosphorylation, and GRP78 and GADD34 expression. J. Neurochem 88, 136-147 (2004)

27. Inagi, R. et al. Preconditioning with endoplasmic reticulum stress ameliorates mesangioproliferative glomerulonephritis. J. Am. Soc. Nephrol. 19, 915-922 (2008).

28. Calabrese, E. J. Hormesis and risk assessment. Oxidat Stress Dis. 34, 339-355 (2014).

29. Mao, X. R. \& Crowder, C. M. Protein misfolding induces hypoxic preconditioning via a subset of the unfolded protein response machinery. Mol. Cell Biol. 30, 5033-5042 (2010).
30. Li, J., Lai, X., Chen, Y., Niu, B. \& Gong, J. Endotoxin tolerance attenuates liver ischemia/reperfusion injury by down-regulation of interleukin-1 receptorassociated kinase 4 in Kupffer cells. Transplant. Proc. 43, 2531-2535 (2011).

31. Juhl, M. et al. Physical exercise during pregnancy and the risk of preterm birth: a study within the Danish National Birth Cohort. Am. J. Epidemiol. 167, 859-866 (2008).

32. $\mathrm{Wu}$, J. et al. The unfolded protein response mediates adaptation to exercise in skeletal muscle through a PGC-1alpha/ATF6alpha complex. Cell Metab. 13 160-169 (2011).

33. Adams Waldorf, K. et al. Uterine overdistention induces preterm labor mediated by inflammation: observations in pregnant women and nonhuman primates. Am. J. Obstet. Gynecol. 213, 830 e831-830 e819 (2015).

34. Atsumi, G. et al. Fas-induced arachidonic acid release is mediated by Ca2 +-independent phospholipase A2 but not cytosolic phospholipase A2, which undergoes proteolytic inactivation. J. Biol. Chem. 273, 13870-13877 (1998).

35. Gross, G. A. et al. Opposing actions of prostaglandins and oxytocin determine the onset of murine labor. Proc. Natl Acad. Sci. USA 95, 11875-11879 (1998).

36. Stocco, C. O. \& Deis, R. P. Participation of intraluteal progesterone and prostaglandin F2 alpha in LH-induced luteolysis in pregnant rat. J. Endocrinol. 156 253-259 (1998). 